\title{
Aberrant expression of hSef and Sprouty 4 in endometrial adenocarcinoma
}

\author{
HUI ZHANG ${ }^{1}$, QIUFEN GUO $^{1}$, XIA WANG $^{2}, \mathrm{CHONG}$ WANG $^{3}$, XINGBO ZHAO $^{1}$ and MINGJIANG LI ${ }^{1}$ \\ ${ }^{1}$ Department of Obstetrics and Gynecology, Shandong Provincial Hospital Affiliated to Shandong University, Jinan, \\ Shandong 250021; Departments of ${ }^{2}$ Nursing and ${ }^{3}$ General Surgery, Shandong Rongjun General Hospital, \\ Jinan, Shandong 250013, P.R. China
}

Received August 28, 2014; Accepted June 16, 2015

DOI: $10.3892 / \mathrm{ol} .2015 .3835$

\begin{abstract}
Fibroblast growth factor (FGF) 2-mediated signaling of the mitogen-activated protein kinase/RAS/extracellular signal-regulated kinase $1 / 2$ pathway is a critical modulator in angiogenesis and is therefore essential for the pathogenesis of endometrial carcinoma. Human similar expression to FGFs (hSef) and Sprouty4 have each been reported to be negative regulators of FGF signaling. The aim of the present study was to investigate the expression of hSef and Sprouty4 in human endometrial adenocarcinoma. Using immunohistochemistry analysis, the expression of hSef and Sprouty4 was detected in human endometrial adenocarcinomas. Increased hSef expression was found to be present in endometrial adenocarcinomas. In addition, decreased hSef expression was identified in the blood vessels of endometrial adenocarcinoma samples. However, the expression of Sprouty4 was downregulated in human endometrial adenocarcinoma. Aberrant expression of hSef and Sprouty4 are involved in the pathogenesis of human endometrial adenocarcinoma.
\end{abstract}

\section{Introduction}

Fibroblast growth factor (FGF) was one of the first confirmed angiogenesis-associated growth factors (1) that play a critical role in the neovascularization of various solid tumors (2-5). By binding to FGF receptors, FGF2 activates the mitogen-activated protein kinase (MAPK) pathway $(6,7)$. As a characteristic MAPK, extracellular signal-regulated kinase 1/2 (ERK1/2) plays a central role in mitogenic signaling, and activated ERK1/2 triggers a series of responses in target cells, including the proliferation and migration of cells (8).

Correspondence to: Professor Mingjiang Li, Department of Obstetrics and Gynecology, Shandong Provincial Hospital Affiliated to Shandong University, 324 Jingwu Road, Jinan, Shandong 250021, P.R. China

E-mail: limingjiang1963@126.com

Key words: hSef, Sprouty4, endometrial adenocarcinoma, fibroblast growth factor 2/mitogen-activated protein kinase/extracellular signal-regulated kinase $1 / 2$ signaling, blood vessel
Similar expression to FGF (hSef) is a negative feedback regulator ofFGF-mediated MAPK/ERK1/2 signaling (9-11) and exerts an inhibitory function by affecting the FGF signaling cascade at multiple levels (9). Sprouty genes have been reported to act as an additional negative feedback regulator of FGF signaling (12) by interrupting the interaction of the growth factor receptor-bound protein $2 /$ son of sevenless complex with fibroblast growth factor receptor substrate 2 and Src homology 2 domain-containing tyrosine phosphatase $(13,14)$, preventing Raf activation $(15,16)$.

Aberrant FGF signaling has been identified in human endometrial carcinoma, and this aberrant expression facilitates the growth and invasion of cancer cells (17). In a previous study, hSef was identified as a negative feedback regulator of FGF-mediated MAPK/ERK1/2 signaling in endometrial cancer cells (11). Plasmid-driven hSef expression has also been revealed to significantly downregulate the growth of endometrial carcinoma Ishikawa cells (11). However, the expression pattern of these negative regulators in endometrial cancers has not yet been investigated. In the current study, the aberrant expression of hSef and Sprouty4 was explored in endometrial adenocarcinoma.

\section{Materials and methods}

Tissue collection and immunohistochemical analysis. In the present study, immunohistochemical analysis was performed on the normal tissue samples obtained from endometrial biopsies performed on 27 women of reproductive age at Shandong Provincial Hospital Affiliated to Shandong University (Jinan, China), with 15 tissues excised in the proliferative phase and 13 in the secretory phase, and cancer tissues obtained from 28 patients with endometrial adenocarcinoma. The diagnosis of endometrial adenocarcinoma was confirmed by histological examination. None of the participants received any hormonal therapy throughout the 3 months prior to the surgical procedure. The present study was approved by the Institutional Review Board of Shandong Provincial Hospital Affiliated to Shandong University and written informed consent was obtained from all participants.

The immunohistochemical analysis was performed as previously described (18). Briefly, the fresh tissues were washed with phosphate-buffered saline (PBS) and then fixed in 4\% paraformaldehyde. Subsequent to dehydration and paraffin-embedding, the samples were cut into 5- $\mu \mathrm{m}$ sections and mounted onto glass 

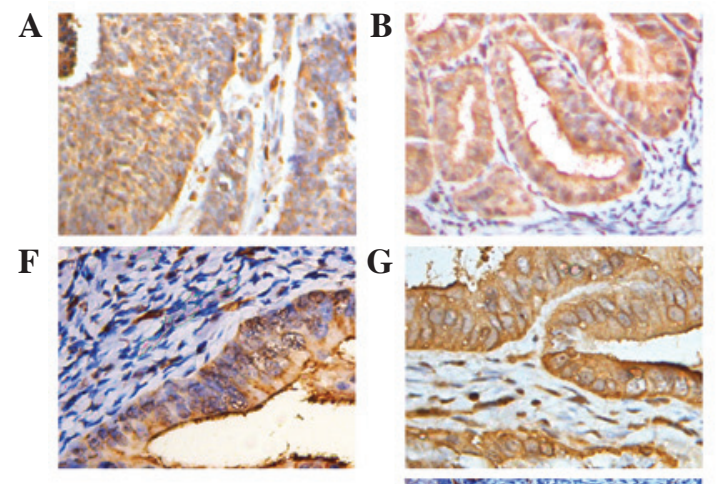

$\mathbf{a}$

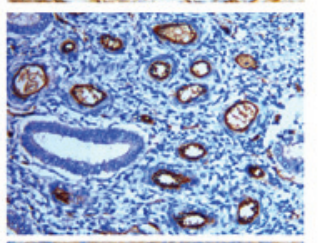

d

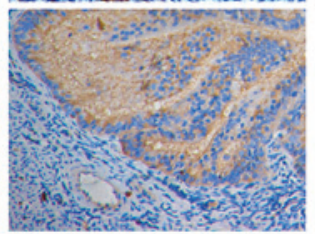

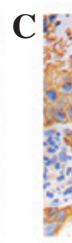
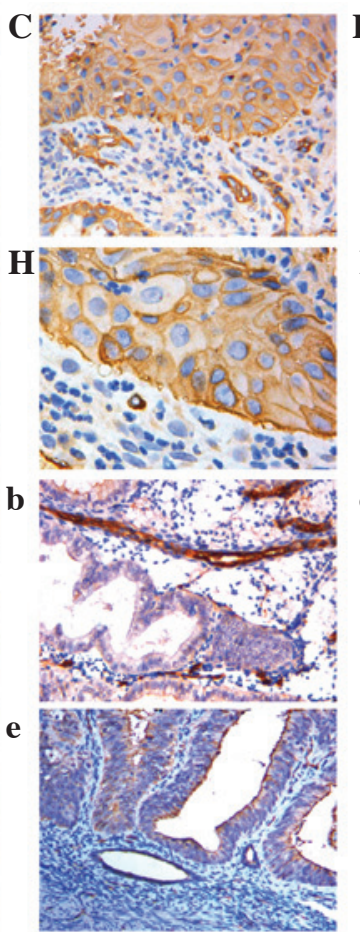
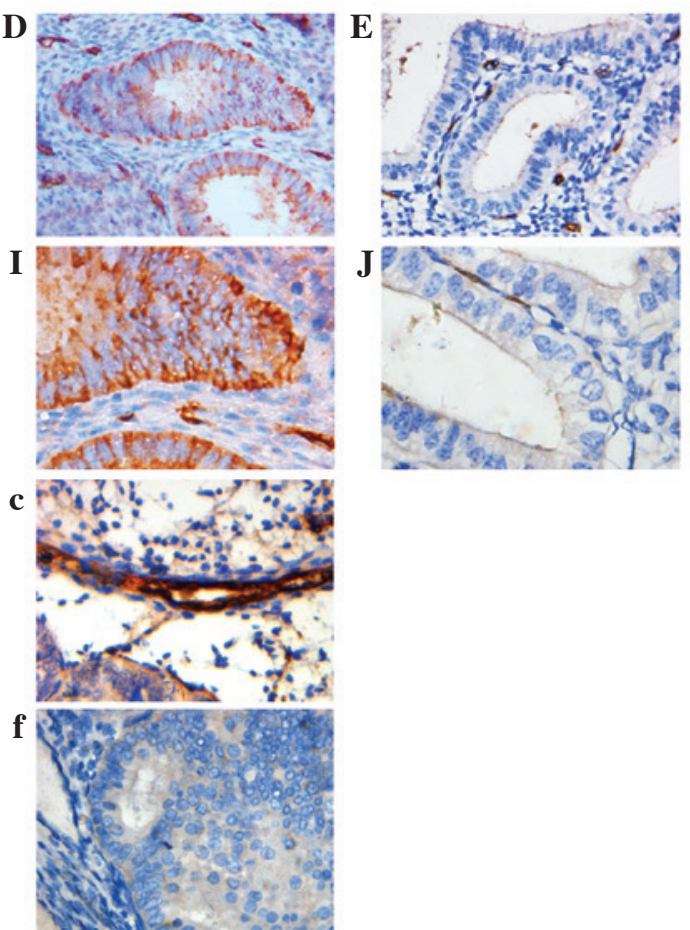

Figure 1. hSef expression in endometrial cancer. Increased hSef expression was observed in endometrial adenocarcinoma, while decreased hSef expression was observed in the vascular endothelial cells of endometrial adenocarcinoma. Immunohistochemical analysis of the expression of hSef in (A-C) endometrial adenocarcinoma and (D and E) normal endometrial tissues, observed at a magnification of x200. Expression of hSef in (F-H) endometrial carcinoma and (I and J) normal endometrial tissue, observed at a magnification of $\mathrm{x} 400$. Expression of hSef in the blood vessels of normal endometrial tissues at a magnification of (a and b) x200 and (c) x400, and in the blood vessels of endometrial adenocarcinoma tissues at a magnification of (d and e) x200 and (f) x400 was also determined using immunohistochemical analysis. Each experiment was performed in duplicate. hSef, human similar expression to fibroblast growth factors.

slides. Deparaffinized and rehydrated sections were incubated with $100 \mu 13 \% \mathrm{H}_{2} \mathrm{O}_{2}$ for $10 \mathrm{~min}$ at room temperature and antigen retrieval was performed. Subsequent to blocking, the sections were incubated overnight with goat anti-human hSef primary polyclonal antibody diluted in phosphate-buffered saline (PBS; dilution, 1:100; cat no. AF2275; R\&D Systems, Inc., Minneapolis, MN, USA) or rabbit anti-human Sprouty4 primary monoclonal antibody diluted in PBS (dilution, 1:100; cat no. ab103114; Abcam, Cambridge, UK) overnight in a wet chamber at $4^{\circ} \mathrm{C}$. Horseradish peroxidase-conjugated rabbit anti-goat or goat anti-rabbit IgG was used as secondary antibody. Tissue sections incubated with non-immune serum instead of primary antibody were used as a negative control. The experiments were repeated in duplicate or triplicate.

The immunohistochemical score was evaluated as previously described (18). Two sections per sample were evaluated for immunohistochemistry in a blind manner, without any knowledge of the clinical or pathological data.

Statistical analysis. Statistical analyses were performed using SPSS software, version 11.5 (SPSS, Inc., Chicago, IL, USA). The data were expressed as the mean \pm standard deviation. Differences between two and multiple groups were determined by one-way analysis of variance. $\mathrm{P}<0.05$ was considered to indicate a statistically significant difference.

\section{Results}

Expression of hSef is upregulated in human endometrial adenocarcinoma. The endothelial expression of hSef in human endometrial adenocarcinoma was determined using immunohistochemical analysis. The immunostaining of hSef in endometrial adenocarcinoma tissues was strong and confined to the cytoplasm of the cancer cells (Fig. 1A-C and F-H). As shown in Fig. 1, in the normal endometrium, the immunostaining of hSef was present or null and mostly confined to the cytoplasm of epithelial cells (Fig. 1D, E, I and J). However, no significant difference was identified between the endometrial tissue samples in the proliferative and secretory phases (Fig. $2 \mathrm{~B}$; $\mathrm{P}>0.05$ ). Compared with normal endometrial tissue, endometrial carcinoma demonstrated increased hSef expression (Fig. 2A, C and D; P<0.05). These data suggest the possible role of hSef overexpression in the pathogenesis and development of human endometrial adenocarcinoma.

Expression of hSef is downregulated in the blood vessels of human endometrial adenocarcinoma tissues. During the immunohistochemical analysis of hSef expression in endometrial carcinoma, the blood vessels in endometrial carcinoma tissues (Fig. 1D-F) were found to exhibit decreased hSef expression compared with the expression of hSef in normal endometrial tissue (Figs. $1 \mathrm{~A}-\mathrm{C}$ and 3 ; $\mathrm{P}<0.05$ ). No significant difference was identified between the hSef expression in endometrial tissue in the proliferative phase and endometrial tissue in the secretory phase (Fig. 3B; P>0.05). These data indicate the possible role of the downregulation of hSef expression in blood vessels in the pathogenesis and development of human endometrial adenocarcinoma.

Loss of Sprouty4 expression occurs in human endometrial adenocarcinoma. The expression of Sprouty4, another 
Immunoscore by immunohistochemistry analysis

A

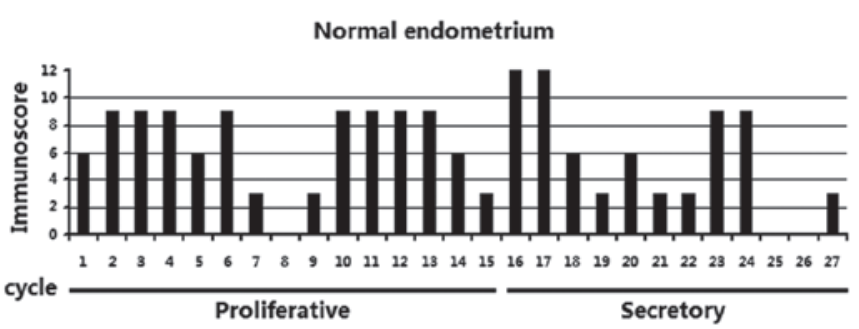

$\mathbf{C}$

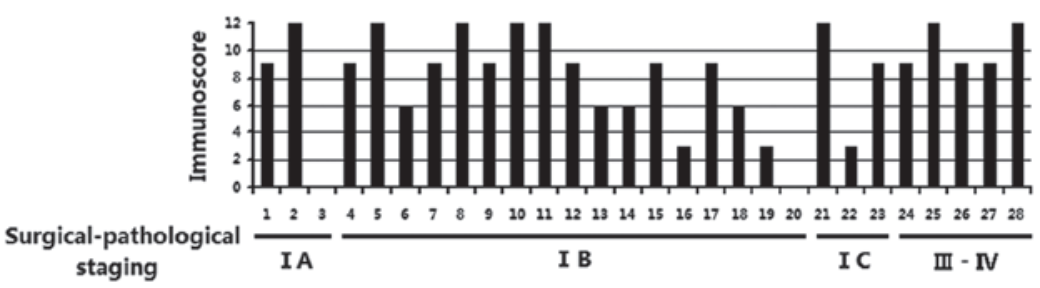

B

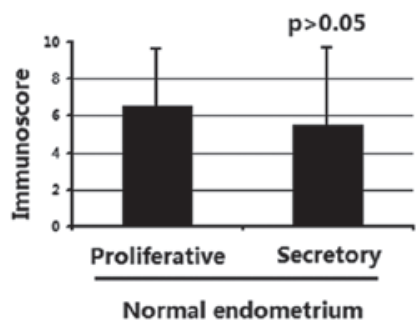

D

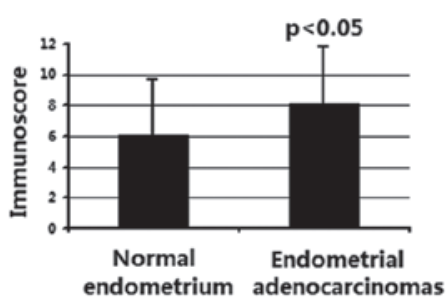

Figure 2. Immunoscore of hSef expression in endometrial cancer tissue. Increased hSef expression was identified in endometrial adenocarcinoma. (A) Detailed data and (B) mean $\pm \mathrm{SD}$ of the immunoscore for hSef expression in normal endometrial tissue in the proliferative and secretory phases. (C) Detailed data and (D) mean $\pm \mathrm{SD}$ of the immunoscore for hSef expression in endometrial adenocarcinoma tissue in the surgical-pathological stage. Each experiment was performed in duplicate. SD, standard deviation; hSef, human similar expression to fibroblast growth factors.

\section{Immunoscore by immunohistochemistry analysis}

A

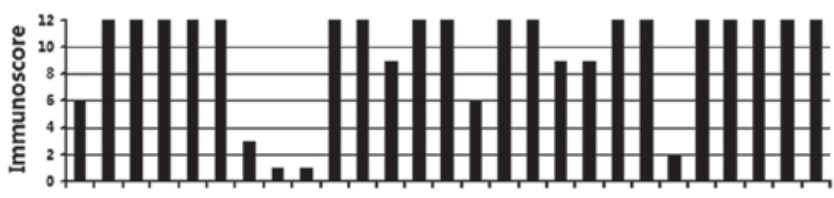

Menstrual cycle

123

Proliferative

Normal endometrium
C

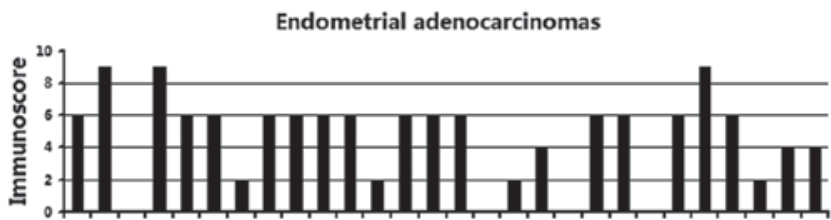

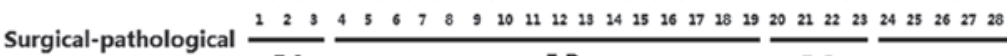

B

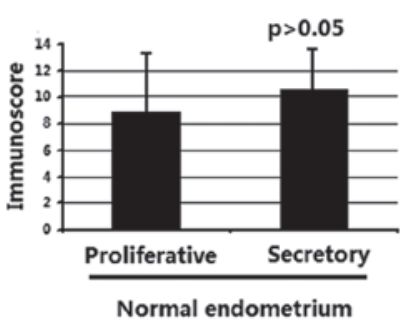

D

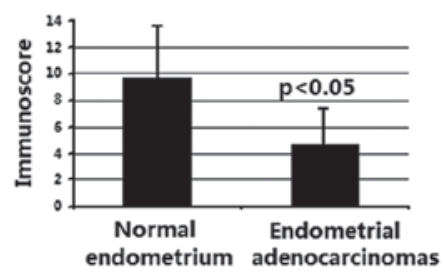

Figure 3. Decreased hSef expression was observed in the vascular endothelial cells of endometrial cancer tissue. (A) Detailed data and (B) mean \pm SD of the immunoscore for hSef expressionin the blood vessels of normal endometrial tissue in the proliferative and secretory phases. (C) Detailed data and (D) mean $\pm \mathrm{SD}$ of the immunoscore for hSef expression in the blood vessels of endometrial adenocarcinoma tissue in the surgical-pathological stage. Each experiment was performed in duplicate. hSef, human similar expression to fibroblast growth factors; SD, standard deviation.

negative regulator of FGF signaling, was also detected in human endometrial carcinoma tissue samples. To determine the expression of Sprouty4 in human endometrial adenocarcinoma, immunohistochemical analysis was performed. Endometrial cancer tissue samples obtained from 31 patients with endometrial adenocarcinoma were used in the analysis. Normal endometrial tissue samples obtained from 30 women of reproductive age were used as a control. As demonstrated by the immunohistochemical analysis results, the immunostaining for Sprouty4 was strong and constricted to the cytoplasm of glandular cells in normal endometrial tissues (Fig. 4A, B, E and F). In endometrial adenocarcinoma tissues, the staining for Sprouty4 revealed absent or weak expression, either in the cancer tissues or 

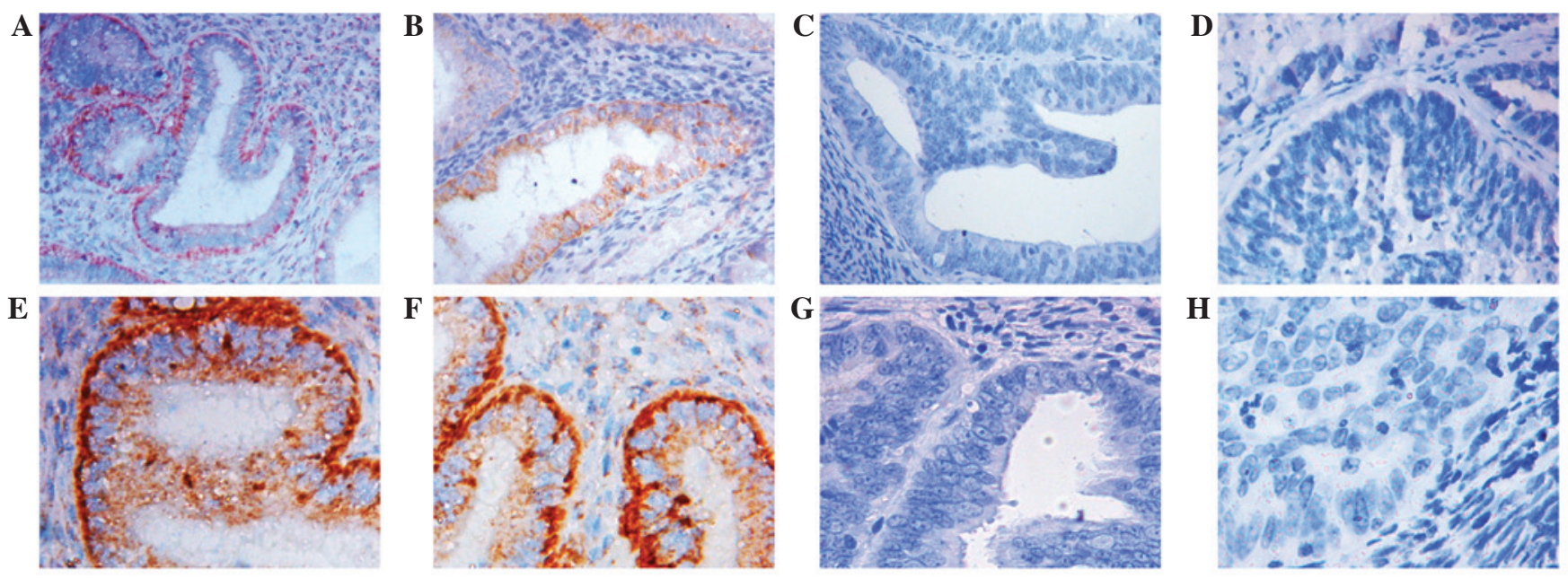

Figure 4. Immunohistochemical analysis of Sprouty4 expression in endometrial cancer tissues. Decreased expression of Sprouty4 was identified in endometrial cancer tissues compared with normal endometrium. The expression of Sprouty4 in (A and B) normal endometrial and (C and D) endometrial adenocarcinoma tissues, observed at a magnification of x200. The expression of Sprouty4 in (E and F) normal endometrial and $(\mathrm{G}$ and $\mathrm{H})$ endometrial adenocarcinoma tissues, observed at a magnification of $\mathrm{x} 400$.

A

Immunoscore by immunohistochemistry analysis
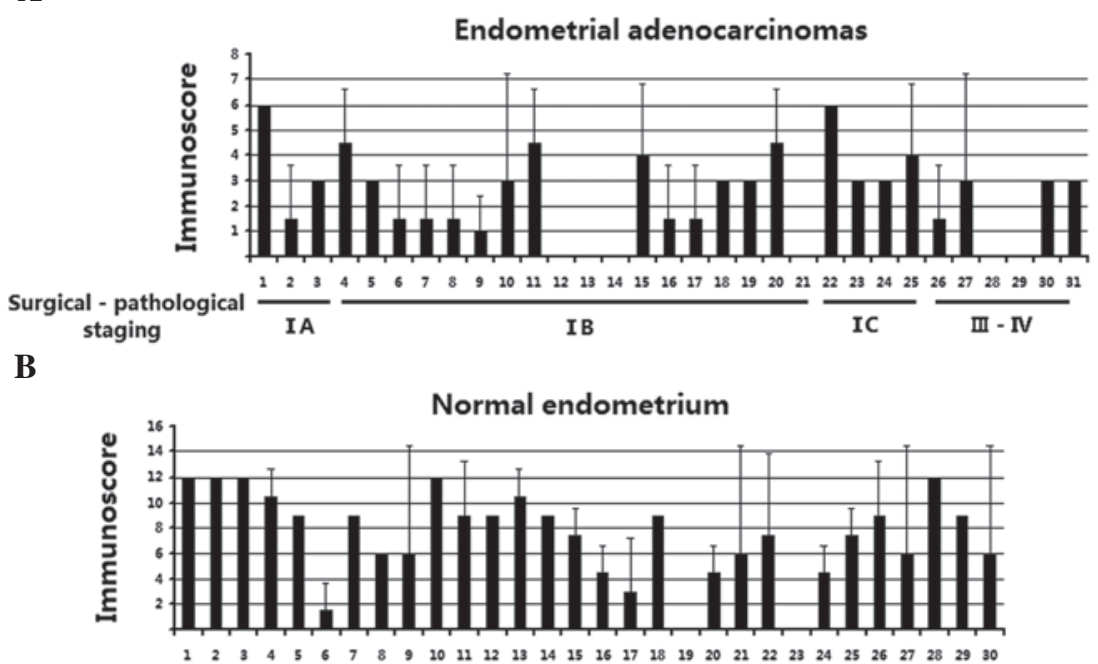

C

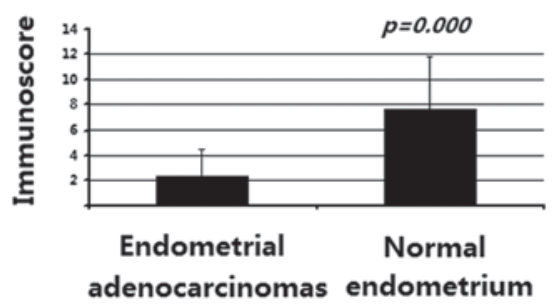

Figure 5. Decreased Sprouty4 expression was identified in endometrial cancer tissues compared with normal endometrial tissues. (A) Detailed data of the immunoscore for Sprouty4 expression in endometrial adenocarcinoma and (B) normal endometrial tissues. (A) The surgical-pathological stage of endometrial carcinomas is reported. (C) The immunoscore data expressed as the mean \pm standard deviation. Each experiment was performed in duplicate.

the stroma (Fig. 4C, D, G and H). The detailed immunostaining scores are reported in Fig. 5. As shown in Fig. 5, the Sprouty4 immunostaining was significantly decreased in endometrial adenocarcinoma tissues $(\mathrm{P}<0.01)$. These data suggest that the loss of Sprouty4 expression is involved in the pathogenesis and development of human endometrial adenocarcinoma.

\section{Discussion}

Abnormal angiogenesis has been reported to participate in the pathogenesis of human endometrial carcinoma, which is one of the most common malignancies of the female genital tract (19). FGF signaling is closely associated with neovascularization in numerous human carcinomas (2-5). In the present study, hSef 
expression was observed to be upregulated in endometrial carcinoma tissues, while the expression of Sprouty4 was downregulated in these tissues. However, the blood vessels in these tissue specimens exhibited decreased hSef expression.

As a negative feedback regulator of FGF/MAPK signaling, hSef exerts an inhibitory effect by acting on multiple points of the FGF/RAS/MAPK cascade. hSef has been found to inhibit RAS (20) and FGF-induced phosphorylation of FGF receptors (20), and block the nuclear translocation of activated ERK1/2 (21). hSef expression is induced by FGF signaling (11), and therefore, hSef is a negative feedback mechanism and self-restricting element of FGF signaling. Activated MAPK/ERK1/2 signaling is involved in caryomitosis, gene expression, and cell proliferation and survival, which indicates that FGF/MAPK signaling is significant in the genesis and development of tumors.

FGF-mediated signaling is involved in the pathogenesis and development of endometrial carcinoma (17). Elevated FGF expression and secretion have been reported to be present in endometrial cancers (17). Plasmid-driven overexpression of FGF in endometrial adenocarcinoma cells has been revealed to promote the formation and growth of tumors in nude mice (22), while administration of the FGF antibody attenuates this process (23). Thus, as a negative feedback regulator of FGF signaling, hSef plays an important role in the pathogenesis of carcinoma. The loss of hSef expression may lead to over-activated FGF signaling, and therefore, to the development of endometrial carcinoma.

Loss of hSef expression is considered to be a common mechanism in epithelial neoplasia. Decreased hSef expression was identified in prostate cancer cells and advanced prostate cancer, and siRNA-mediated hSef downregulation enhanced FGF-induced cell migration and invasion in prostate cancer cells $(24,25)$. In in vitro studies, hSef overexpression was found to inhibit cell proliferation, migration and invasive potential in prostate cancer cells (26). Loss of hSef expression was also observed in breast, thyroid and ovarian carcinomas. The downregulation of hSef expression was found to be associated with tumor progression (27). In addition, ectopic hSef expression reduced the growth of breast cancer cells, while inhibition of hSef expression accelerated FGF-induced growth in cervical cancer cells (27).

In the current study, an unexpected increase in hSef expression was observed in endometrial adenocarcinomas. As hSef is inducible by FGF-mediated MAPK signaling, the upregulation of hSef expression may be due to the elevated activity of MAPK. In previous studies, plasmid-driven hSef expression has been demonstrated to reduce FGF2-mediated MAPK/ERK signaling and cell growth in Ishikawa cells, a well-differentiated endometrial adenocarcinoma cell line (11). This indicates that hSef may function as a tumor suppresser in endometrial cancer cells. However, considering the current study, it is hypothesized that hSef may be also involved in other mechanisms. These data indicate the potential roles of hSef in the pathogenesis of endometrial carcinomas.

In the present study, the blood vessels in endometrial cancer tissues were also found to demonstrate decreased hSef expression. The human endometrium undergoes cyclic changes of degradation and reestablishment, which results in angiogenesis being of considerable significance in the physiology and carcinogenesis of the endometrium. Considering the critical role of FGF signaling in the neovascularization of various solid tumors (2-5), the loss of hSef expression may facilitate the activity of FGF signaling, subsequently promoting the neovascularization in endometrial cancers. The potential role of hSef in the neovascularization of tumors requires additional investigation.

The loss of Sprouty expression has been identified in a series of human carcinomas, including prostate cancer (28-30), breast carcinoma $(31,32)$, hepatocellular cancer $(33,34)$, lung carcinoma (35), melanoma (36), colon cancer (37) and liver cancer (38). In addition, the downregulation of Sprouty 2 has been identified in endometrial carcinoma tissue (39). In the present study, the loss of Sprouty4 expression was also identified in human endometrial adenocarcinoma tissue samples, indicating the possible role of Sprouty4 in endometrial carcinogenesis.

Overall, to the best of our knowledge, the present study demonstrated that increased hSef expression and decreased Sprouty4 are present in endometrial carcinoma tissue, while decreased hSef expression is observed in the blood vessels of endometrial carcinoma. These data indicate the various roles of negative regulators of FGF signaling in the pathology and genesis of human endometrial carcinoma tissues. The present study provides novel insight into the pathogenesis of endometrial cancer, and suggests a potential therapeutic target for gene-target therapy of the disease.

\section{Acknowledgements}

This study was supported by the National Natural Science Foundation of China (grant nos. 81170549 and 81300468), the Shandong Province Excellent Youth Scientist Foundation (grant nos. BS2013YY008 and 2009BSB14147) and the Shandong Natural Science Foundation (grant no. ZR2011HM045).

\section{References}

1. Shing Y, Folkman J, Sullivan R, Butterfield C, Murray J and Klagsbrun M: Heparin-affinity: Purification of a tumor-derived capillary endothelial cell growth factor. Science 223: 1296-1298, 1984.

2. Soulitzis N, Karyotis I, Delakas D and Spandidos DA: Expression analysis of peptide growth factors VEGF, FGF2, TGFB1, EGF and IGF1 in prostate cancer and benign prostatic hyperplasia. Int J Oncol 29: 305-314, 2006.

3. Straume O and Akslen LA: Importance of vascular phenotype by basic fibroblast growth factor and influence of the angiogenic factors basic fibroblast growth factor/fibroblast growth factor receptor-1 and EphA1/EphA2 on melanoma progression. Am J Pathol 160: 1009-1019, 2002.

4. Compagni A, Wilgenbus P, Impagnatiello MA, Cotten M and Christofori G: Fibroblast growth factors are required for efficient tumor angiogenesis. Cancer Res 60: 7163-7169, 2000.

5. Soufla G, Sifakis S, Baritaki S, Zafiropoulos A, Koumantakis E and Spandidos DA: VEGF, FGF2, TGFB1 and TGFBR1 mRNA expression levels correlate with the malignant transformation of the uterine cervix. Cancer Lett 221: 105-118, 2005.

6. Javerzat S, Auguste P and Bikfalvi A: The role of fibroblast growth factors in vascular development. Trends Mol Med 8: 483-489, 2002.

7. Schlessinger J, Plotnikov AN, Ibrahimi OA, Eliseenkova AV, Yeh BK, Yayon A, Linhardt RJ and Mohammadi M: Crystal structure of a ternary FGF-FGFR-heparin complex reveals a dual role for heparin in FGFR binding and dimerization. Mol Cell 6: 743-750, 2000.

8. Pearson G, Robinson F, Beers Gibson T, Xu BE, Karandikar M, Berman K and Cobb MH: Mitogen-activated protein (MAP) kinase pathways: Regulation and physiological functions. Endocr Rev 22: 153-183, 2001. 
9. Thisse B and Thisse C: Functions and regulations of fibroblast growth factor signaling during embryonic development. Dev Biol 287: 390-402, 2005.

10. Tsang M and Dawid IB: Promotion and attenuation of FGF signaling through the Ras-MAPK pathway. Sci STKE 2004: pe17, 2004

11. Zhang H, Zhao X, Yan L and Li M: Similar expression to FGF (Sef) reduces endometrial adenocarcinoma cells proliferation via inhibiting fibroblast growth factor 2-mediated MAPK/ERK signaling pathway. Gynecol Oncol 122: 669-674, 2011.

12. Hacohen N, Kramer S, Sutherland D, Hiromi Y and Krasnow MA: sprouty encodes a novel antagonist of FGF signaling that patterns apical branching of the Drosophila airways. Cell 92: 253-263, 1998.

13. Gross I, Bassit B, Benezra M and Licht JD: Mammalian sprouty proteins inhibit cell growth and differentiation by preventing ras activation. J Biol Chem 276: 46460-46468, 2001.

14. Hanafusa $H$, Torii $S$, Yasunaga T, Matsumoto K and Nishida E: Shp2, an SH2-containing protein-tyrosine phosphatase, positively regulates receptor tyrosine kinase signaling by dephosphorylating and inactivating the inhibitor Sprouty. J Biol Chem 279: 22992-22995, 2004.

15. Sasaki A, Taketomi T, Kato R, Saeki K, Nonami A, Sasaki M, Kuriyama M, Saito N, Shibuya M and Yoshimura A: Mammalian SPRY4 suppresses Ras-independent ERK activation by binding to Raf1. Nat Cell Biol 5: 427-432, 2003.

16. Yusoff P, Lao DH, Ong SH, Wong ES, Lim J, Lo TL, Leong HF, Fong CW and Guy GR: Sprouty2 inhibits the Ras/MAP kinase pathway by inhibiting the activation of Raf. J Biol Chem 277: 3195-3201, 2002.

17. Billottet C, Elkhatib N, Thiery JP and Jouanneau J: Targets of fibroblast growth factor 1 (FGF-1) and FGF-2 signaling involved in the invasive and tumourigenic behavior of carcinoma cells. Mol Biol Cell 15: 4725-4734, 2004

18. Zhang H, Li M, Zheng X, Sun Y, Wen Z and Zhao X: Endometriotic stromal cells lose the ability to regulate cell-survival signaling in endometrial epithelial cells in vitro. Mol Hum Reprod 15: 653-663, 2009.

19. Abulafia O, Triest WE and Sherer DM: Angiogenesis in\&\#12288; malignancies of the female genital tract. Gynecol Oncol 72: 220-231, 1999.

20. Macdonald SG, Crews CM, Wu L, Driller J, Clark R, Erikson RL and McCormick F: Reconstitution of the Raf-1-MEK-ERK signal transduction pathway in vitro. Mol Cell Biol 13: 6615-6620, 1993.

21. Ballif BA and Blenis J: Molecular mechanisms mediating mammalian mitogen-activated protein kinase (MAPK) kinase (MEK)-MAPK cell survival signals. Cell Growth Differ 12: 397-408, 2001.

22. Giavazzi R, Sennino B, Coltrini D, Garofalo A, Dossi R, Ronca R, Tosatti MP and Presta M: Distinct role of fibroblast growth factor-2 and vascular endothelial growth factor on tumour growth and angiogenesis. Am J Pathol 162: 1913-1926, 2013.

23. Hori A, Sasada R, Matsutani E, Naito K, Sakura Y, Fujita T and Kozai Y: Suppression of solid tumor growth by immunoneutralizing monoclonal antibody against human basic fibroblast growth factor. Cancer Res 51: 6180-6184, 1991.

24. Darby S, Sahadevan K, Khan MM, Robson CN, Leung HY and Gnanapragasam VJ: Loss of Sef (similar expression to FGF) expression is associated with high grade and metastatic prostate cancer. Oncogene 25: 4122-4127, 2006.

25. Murphy T, Darby S, Mathers ME and Gnanapragasam VJ: Evidence for distinct alterations in the FGF axis in prostate cancer progression to an aggressive clinical phenotype. J Pathol 220: 452-460, 2010.
26. Darby S, Murphy T, Thomas H, Robson CN, Leung HY, Mathers ME and Gnanapragasam VJ: Similar expression to FGF (Sef) inhibits fibroblast growth factor-induced tumourigenic behaviour in prostate cancer cells and is downregulated in aggressive clinical disease. Br J Cancer 101: 1891-1899, 2009.

27. Zisman-Rozen S, Fink D, Ben-Izhak O, Fuchs Y, Brodski A, Kraus MH, Bejar J and Ron D: Downregulation of Sef, an inhibitor of receptor tyrosine kinase signaling, is common to a variety of human carcinomas. Oncogene 26: 6093-6098, 2007.

28. Wang J, Thompson B, Ren C, Ittmann M and Kwabi-Addo B: Sprouty4, a suppressor of tumor cell motility, is down regulated by DNA methylation in human prostate cancer. Prostate 66: 613-624, 2006.

29. Kwabi-Addo B, Wang J, Erdem H, Vaid A, Castro P, Ayala G and Ittmann M: The expression of Sprouty1, an inhibitor of fibroblast growth factor signal transduction, is decreased in human prostate cancer. Cancer Res 64: 4728-4735, 2004.

30. Fritzsche S, Kenzelmann M, Hoffmann MJ, Müller M, Engers R, Gröne HJ and Schulz WA: Concomitant down-regulation of SPRY1 and SPRY2 in prostate carcinoma. Endocr Relat Cancer 13: 839-849. 2006.

31. Lo TL, Yusoff P, Fong CW, McCaw BJ, Phillips WA, Yang $\mathrm{H}$, Wong ES, Leong HF, Zeng Q, Putti TC, et al: The ras/mitogen-activated protein kinase pathway inhibitor and likely tumor suppressor proteins, sprouty 1 and sprouty 2 are deregulated in breast cancer. Cancer Res 64: 6127-6136, 2004.

32. Faratian D, Sims AH, Mullen P, Kay C, Um I, Langdon SP and Harrison DJ: Sprouty 2 is an independent prognostic factor in breast cancer and may be useful in stratifying patients for trastuzumab therapy. PLoS One 6: e23772, 2011.

33. Fong CW, Chua MS, McKie AB, Ling SH, Mason V, Li R, Yusoff P, Lo TL, Leung HY, So SK, et al: Sprouty 2, an inhibitor of mitogen-activated protein kinase signaling, is down-regulated in hepatocellular carcinoma. Cancer Res 66: 2048-2058, 2006.

34. Sirivatanauksorn Y, Sirivatanauksorn V, Srisawat C, Khongmanee A and Tongkham C: Differential expression of sprouty genes in hepatocellular carcinoma. J Surg Oncol 105: 273-276, 2012

35. Sutterlüty H, Mayer CE, Setinek U, Attems J, Ovtcharov S, Mikula M, Mikulits W, Micksche $\mathrm{M}$ and Berger W: Down-regulation of Sprouty2 in non-small cell lung cancer contributes to tumor malignancy via extracellular signal-regulated kinase pathway-dependent and-independent mechanisms. Mol Cancer Res 5: 509-520, 2007.

36. Qi J, Nakayama K, Gaitonde S, Krajewski S, Eroshkin A, Bar-Sagi D, Bowtell D and Ronai Z: The ubiquitin ligase Siah2 regulates tumorigenesis and metastasis by HIF-dependent and -independent pathways. Proc Natl Acad Sci USA 105: 16713-16781, 2008.

37. Feng YH, Wu CL, Tsao CJ, Chang JG, Lu PJ, Yeh KT, Uen YH, Lee JC and Shiau AL: Deregulated expression of sprouty 2 and microRNA-21 in human colon cancer: Correlation with the clinical stage of the disease. Cancer Biol Ther 11: 111-121, 2011.

38. Lee SA, Ho C, Roy R, Kosinski C, Patil MA, Tward AD, Fridlyand $\mathrm{J}$ and $\mathrm{Chen} \mathrm{X}$ : Integration of genomic analysis and in vivo transfection to identify sprouty 2 as a candidate tumor suppressor in liver cancer. Hepatology 47: 1200-1210, 2008.

39. Velasco A, Pallares J, Santacana M, Gatius S, Fernandez M, Domingo M, Valls J, Yeramian A, Encinas M, Dolcet X, et al: Promoter hypermethylation and expression of sprouty 2 in endometrial carcinoma. Hum Pathol 42: 185-193, 2011. 